\title{
Evidence-based health policymaking in Iraqi Kurdistan: Facilitators and barriers from the perspectives of policymakers and advisors
}

\begin{abstract}
Sherzad Shabu $* \quad$ Namir G. Al-Tawil * $\quad$ Michael P. Fuller $* * \quad$ Rod Sheaff $* * *$
\section{Abstract}

Background and objective: Evidence from research is underutilized in policy and practice in the majority of developing countries including Iraq. This aim of this study was to assess the role of research in health policy making in Iraqi Kurdistan context and identify the main barriers and facilitators for enhancing such role.

Methods: This cross-sectional survey was carried out between November 2013 and March 2014 in the three governorates of the Iraqi Kurdistan Region, Erbil, Duhok and Sulaimaniyah. The study participants included 10 key health informants and three health advisors. Two types of combined questionnaires for health policy makers and health advisors were used for data collection.

Results: Conferences and seminars were the main sources of scientific evidences identified by health policymakers $(80 \%)$, followed by consultants $(70 \%)$. Different jargons/ discourse was the main obstacle in consulting researchers $(90 \%)$, followed by lack of tradition in collaborating (70\%). Collection of specialists/advisors, followed by professional associations, scientific committees and international organizations/UN agencies were the main groups identified by health advisors to build bridges between the scientific community and policymakers.

Conclusion: Policymakers very rarely consult researchers directly in their decision making. There is poor networking among researchers, policy-makers, practitioners and representatives from civil society which has its negative impact on evidence-based policymaking. There is obviously a lack of any sort of program of funded research that can inform policymaking.
\end{abstract}

Keywords: Health policy, Kurdistan region, Erbil, Iraq.

\section{Introduction}

Evidence-based policymaking (EBP) is an approach that "helps people make well informed decisions about policies, programmes and projects by putting the best available evidence from research at the heart of policy development and implementation". ${ }^{1}$ EBP is a discourse or set of methods which informs the policy process, rather than one which aims directly to affect the eventual goals of the policy. It supports a more rational, rigorous and systematic approach of policy-making. The concept of EBP is based on the fact that policy decisions should be better informed by available evidence, and should include rational analysis. This is because policy and practice, which are based on systematic evidence, are seen to produce better outcomes. Therefore, opinion based policies are being replaced by a more rigorous, rational approach that gathers, critically appraises and uses high quality research evidence to inform policymaking and professional practice. ${ }^{2}$ The literature shows that there is a complex relationship between knowledge production (research that produces evidence) and knowledge utilisation (evidence used in policy making, programme implementation, programme

* Department of community medicine, college of medicine, Hawler Medical University, Erbil, Iraq.

** Graduate School, University of Plymouth, UK.

*** School of Government, Faculty of Business, University of Plymouth, UK. 
design, etc.) with the existence of different barriers in front of the use of research in policy. 3,4 Therefore, the process of research utilisation in health care policy making has, in itself, become an important area of study which aims at finding ways to increase the uptake of research findings. ${ }^{5,6}$ The process of policymaking usually involves different stages: agenda setting; formulation; implementation; and evaluation. Research evidence has the potential to influence the policymaking process at each of these stages. The fact is that for different parts of the policymaking process, different types of evidence are required and that the timing factor is likely to influence the mechanisms available to mobilize evidence. ${ }^{2}$ Policymakers may have problems obtaining research evidence, even when available, since they might not have access to the source of such evidence. In addition, some policy makers, mainly those working at the local or country levels, are unable to access the research evidence since they lack the basic information technology skills necessary for this purpose. ${ }^{7}$ A number of other factors might exist which will limit the extent to which research evidence can be utilised in policymaking, like the pressure to produce and process information quickly. In Iraq, including Iraqi Kurdistan, the health system suffers from a lack of system orientation and this has resulted in an excess of policy initiatives, as there are a bits and pieces of project plans than any sort of coordinated strategic planning aligned to key health system goals. ${ }^{8}$ Currently, the Ministries of Health in both Baghdad and Erbil lack clear strategic policy directions and this has led to uncoordinated planning and fragmented projects. As for the majority of developing countries and those countries in postconflict status, Iraq witnessed a poor role of research in health policy development and planning. The reasons for such poor role, whether from the policymakers' side or the researchers' side, are not clearly understood. 9,10 Therefore, this study was carried out to understand and assess the role of research on health policy making in Iraqi Kurdistan context and to identify the main barriers and facilitators for enhancing such role.

\section{Methods}

In Iraqi Kurdistan, the health services, in the public sector, are provided through a network of hospitals and primary health care centers where services are provided with equal chance of access and at very low charges to all people. This is done through a Directorate General of Health in each of the three Iraqi Kurdistan governorates. This cross-sectional survey was carried out between November 2013 and March 2014 in the three governorates of the Iraqi Kurdistan region; Erbil, Duhok and Sulaimaniyah. The study participants included the key health informants within the Ministry of Health in Erbil (the Minister of Health and the Director Generals in addition to the scientific advisors) and the Directorates of Health in Erbil, Duhok and Sulaimaniya (the Director Generals and their Assistants). Twelve key health informants within these health management institutions were selected using the managerial hierarchy in these institutions from the top management level. Two key health informants didn't want to participate in the study. Two separate combined self-administered questionnaires, one for health policymakers and another for health advisors, adapted from the Scientific Evidence for Policy Making, European Research Area, European Commission, ${ }^{11}$ were used for data collection. The questionnaires were sent to participants with a written information sheet explaining the research issue. The questionnaires included questions on the usefulness of research findings in policy making, the main obstacles in consulting researchers, the appropriate bodies that act as intermediaries or build bridges between researchers and policymakers, whether the independence of research should be safeguarded, whether there is a gap 
http://dx.doi.org/10.15218/zjms.2015.0035

of collaboration between the scientific community and policymakers, the need for a program of funded research to inform policymakers, etc. Statistical analysis included descriptive statistics with calculation of frequencies. The study was approved by the Research Ethics Committee of the College of Medicine of Hawler Medical University. Informed consent of study participants was obtained and their anonymity was preserved.

\section{Results}

\section{Evidence-based policymaking from the policymakers' perspectives}

The majority (8 out of 10) of the policymakers have identified conferences and seminars as the main sources of getting scientific evidences for their planning and policy making purposes, followed by consultants $(n=7)$ both national and international (e.g. from USA, UK, Turkey, Sweden, Italy, Germany and those from relevant UN agencies), both academic journals and general press $(n=5)$, and finally professional researchers and lobby organizations $(n=4)$, as shown in Figure 1. All policymakers confirmed that they consider scientific evidence useful for policy decisions; nine of them stated that it is more useful to have it at the stage when they define the policy compared to seven of them who thought it is useful at the stage of evaluation of policy choices. Only two of the policymakers stated that they consult researchers directly in their decision making, and that is done through meetings, workshops and email exchanges. Of 10 policymakers, nine have identified different jargons/discourse as the main obstacle to consulting researchers, followed by lack of tradition in collaborating (7), lack of communication channels and timing on communication (5), and legal barriers (1), as it is shown in Figure 2.

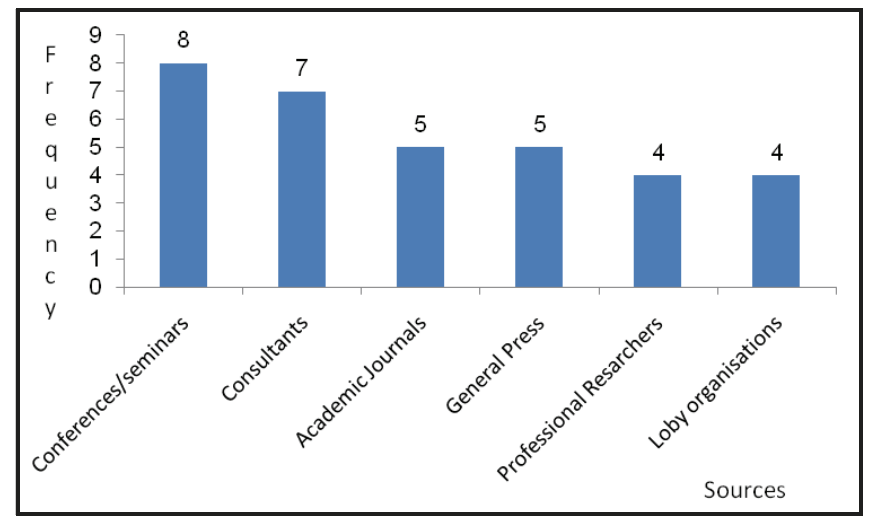

Figure 1: Main sources of scientific evidence.

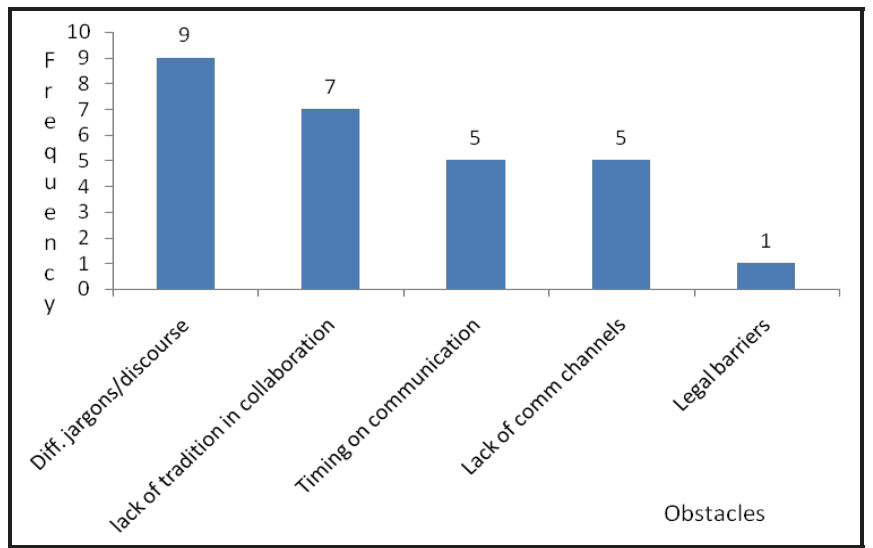

Figure 2: Obstacles to consulting researchers. 
http://dx.doi.org/10.15218/zjms.2015.0035

Most participants (7) disagreed that scientific evidence could be directly transmissible to policymakers. With regard to the most appropriate bodies to act as an intermediary between researchers and policy-makers, nine participants stated that consultants could play this role, followed by collection of specialists/advisors and scientific committees $(n=7)$, professional associations $(n=6)$, international nongovernmental organizations (NGOs)/UN agencies and specialists in knowledge transfer $(n=4)$, and finally NGOs and civil society organizations (CSO) ( $n=3)$, as shown in Figure 3. Nine participants agreed that policymakers should declare their interest or their agenda for priority research topics at the start of research projects.

All the participants stressed the fact that the independence of research (choice of area or methodology) should be safeguarded. Below are examples of some of their quotations on how this could be achieved:

${ }^{*}$ Research to be approved by scientific committees.

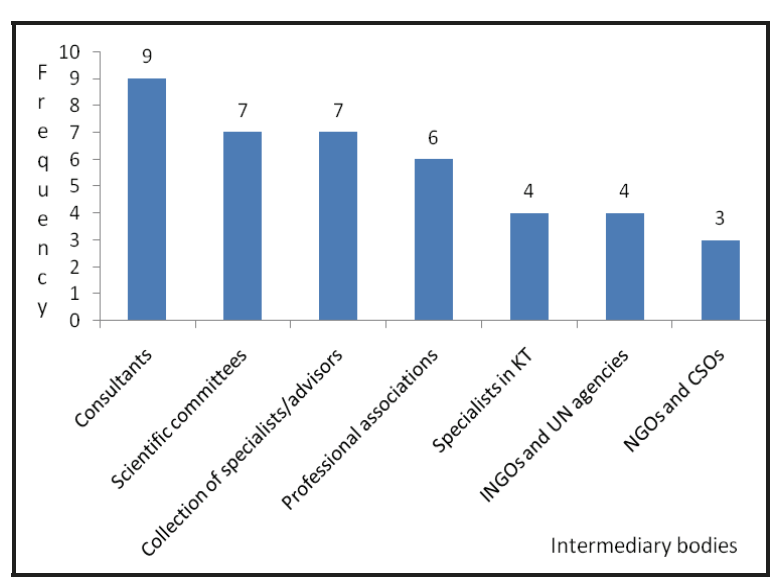

Figure 3: Intermediary bodies between researchers and policymakers as defined by policymakers
*Ethical consideration and follow up by supervisors.

*Provide facilities and minimize influence.

${ }^{*}$ Choose professional and experienced researchers who are not influenced by outside pressure.

*Enhance a culture of non-interference and convince politicians not to interfere.

*Known and tested methodologies, well discussed and shared with policymakers at the planning stage.

*Clear nemorandum of understanding between the scientific body and the relevant authorities.

2. Evidence-based policymaking from the health advisors' perspectives

As shown in Figure 4, all the three participants stressed that there is a gap of collaboration between the scientific community and policymakers, and they gave a mean rate of $3.6 / 5$ for this gap. The participants identified lack of communication channels as the main problem in this regard, followed by lack of tradition in collaboration, and finally different jargons/discourse and timing of communication (Figure 4).

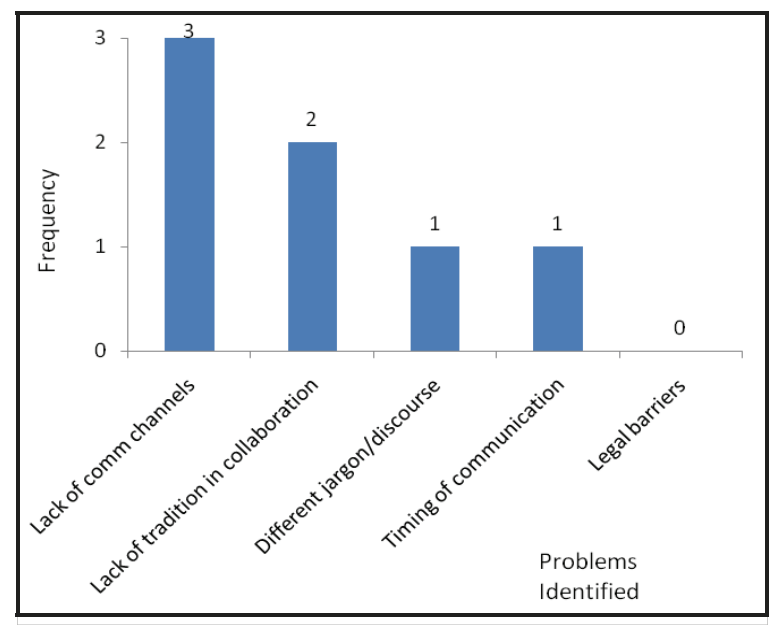

Figure 4: Reasons for collaboration gap between scientific community and policymakers as defined by health advisors 
With regard to the people who could build bridges between the scientific community and policymakers, the participants mentioned collection of specialists/advisors as the main people who can build these bridges, followed by professional associations, scientific committees, international organizations/UN agencies, specialists in knowledge transfer, NGOs and CSOs and finally consultants, as shown in Figure 5. In response to the question on how these gaps could be bridged and how these bridges could be built, below are the quotations from the participants in this regard:

${ }^{*}$ Good planning, scientific needs announced and representatives to meet with the university

*To build a system which guide us in this direction

${ }^{*}$ Regular meetings between both sides, follow up of recommendations and implementations on the ground, hiring of policymakers and advisors to be based on meritocracy not nepotism.

All the participants stressed on that a participative approach - through the creation of networks involving researchers, policy-makers, practitioners and representatives from civil society, could offer the possibility of a continuing cooperation with perspective on action. Below are the participants' explanations on how this could be achieved:

${ }^{*}$ Regular meetings, at least once every two months.

*Through workshops and meetings.

*Brain storming and idea sharing, joint decision making is more sound, realistic and practicable.

The participants also thought that there is a need to have a special body which could be responsible for feeding the scientific evidence to policymakers, and below are their statements on how this can be achieved:

${ }^{*}$ Director General of Health to be a member of the College of Medicine's Council, and the Dean or the University President to be a member of the Directorate of Health Administrative Council.
${ }^{*}$ Representatives from $\mathrm{MoH}$ and University

*To establish a research center.

With regard to a question on whether participants think that there should be a programme of funded research that informs policy making, two of the participants support that with the following explanations:

*This will help in setting up priorities and plan research based on these priorities.

*Financial support guarantee regular research conduction based on real needs; researchers will be more motivated and ready to conduct research without financial constraints, and funded research produces regular information for policymakers

Only one participant did not support this idea and has the following explanation:

*This is not practical because our system is different from the system of universities abroad, here all staff are paid by the government.

All the participants think that the policy making department should have specific scientific advisors on individual issues, and have explained that through the following quotations:

* It will enhance the translation of knowledge into practice.

*In policy making, use of research evidence and strategic planning.

*The Advisor's experience can be helpful in choosing, implementing and supervising the needed research which is relevant to that department, and communicating with all other relevant partners on specific research issue.

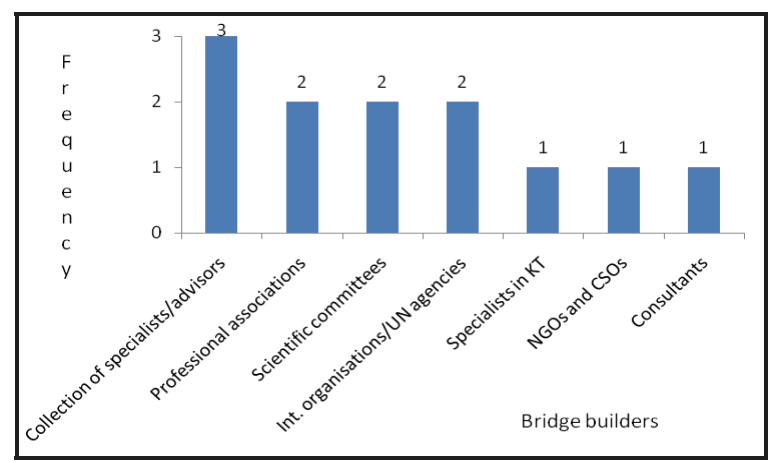

Figure 5: Bridges builders between scientific community and policymakers 


\section{Discussion}

The study revealed that the main sources, where policymakers get their scientific evidence for their planning, were conferences or seminars followed by consultants, while academic journals and professional researchers came at a lesser degree as sources of scientific evidence. Such result reflects the fact of existence of a considerable communication gap between policymakers from one side and academic researchers from another side. These results agree with those from a study conducted in Nigeria which revealed that there is critical gap of communication between policymakers and professional researchers which negatively affect the process of evidence taking for health policymaking. This in addition to the non-availability of research units or departments in most of the health organizations which could be of a great help in presenting relevant academic journals with relevant scientific evidence to policymakers in order to be considered in the policymaking process. ${ }^{12}$ A study conducted in 2001 on how to improve the communication between researchers and policymakers in long-term care revealed that conferences, workshops, and other meetings that are carefully tailored to facilitate communication across the professional divide can be an effective and relatively efficient way to build researcherspolicymakers linkages. ${ }^{13}$ The study also revealed that policymakers are not directly consulting researchers in their decision making and only few participants stated that they do so through meetings, workshops and email exchanges. Similar results were obtained from a study conducted in 2012 to assess the use of health systems and policy research evidence in health policymaking in Eastern Mediterranean countries which revealed that $65 \%$ of the researcher participants agreed that there is lack of co-ordination between policymakers and researchers which hinder the use of evidence in policymaking process, and there is insufficient policy dialogue opportunities, networking and collaboration between these two bodies. ${ }^{14}$ From the perspective of policymakers, the study revealed that the main obstacle in consulting researchers are the different jargons or discourse that have been used by the two groups followed by lack of tradition in communication, lack of communication channels and timing on communication. On the other hand and from the advisors' perspective, lack of communication channels come first followed by lack of tradition in collaboration and then different jargons or discourse and timing on communication. These results agree, to a large extent, with those of a study conducted by Nutley in 2003 which revealed that the limitation of interaction between policymakers and researchers is mainly due to the divergence of the worlds of these two groups since they use different languages and have different agenda, priorities, timescales and reward systems and hence there is always lack of effective communication between the two groups. ${ }^{15}$ In 2011, a research conducted by Hyder et al, in a number of developing countries revealed that policymakers have repeatedly stressed the importance of communication of research results to policymakers and identified communication factor as the most important barrier in this regard since there are no formal channels for research findings to communicated to policymakers. In this study, policymakers also suggested that the lack of so called research culture has played an important role in creating the gap between policymakers and researchers and revealed that the importance of such culture was considered to be a prerequisite for evidence-based health policymaking. ${ }^{160}$ Most of the participants thought that scientific evidence is never directly transmissible to policymakers and that there should be some appropriate intermediary bodies which can work between researchers and policymakers. From the policymakers' perspectives, 
consultants were the most appropriate people to achieve this, followed by scientific committees and collection of specialists/advisors, professional associations, while specialists in knowledge translation, UN agencies, NGOs and CSOs were the least appropriate bodies. From the advisors' perspective, collections of specialists/advisors were the most appropriate bodies followed by professional associations, scientific committees and UN agencies and International NGOs. It is obvious that the priority is for consultants, specialists and advisors but much less demand put on specialist in knowledge translation, since they are simply unavailable yet, and also on CSOs which reflects their relative poor contribution in this regard. A study conducted in a number of low- and middle-income countries in 2011 also revealed that national policymakers recommended having an office composed of specialists who will be able to look at the disseminated research results, critically analyse them and advise policymakers on the appropriate use of these results. ${ }^{16}$ The study also revealed that policymakers should declare their interest or their agenda for priority research topics at the start of research projects so that these research topics are considered by individual researchers or research institutions in their planning. These results agree with those of a review study and synthesis of literature on knowledge transfer and exchange in 2007 which revealed that some of the main knowledge transfer facilitators are involvement of decision makers in research planning and design and emphasizing a collaborative research partnerships between researchers and policymakers. ${ }^{17}$ The results also agree with those of the WHO study conducted in 2005 which stated that one of the common principles showed by different studies conducted to identify the key factors to strengthen the research to action link is that decision makers should participate in setting objectives and formulating the agenda for both research and dissemination activities. $^{18}$ The Advisors stressed that a participative approach, through the creation of networks involving researchers, policymakers, practitioners and representatives from civil society, could offer the possibility of a continuing cooperation with perspective on action. They thought that this can be achieved through regular meetings and workshops. These results are in agreement with those of a study conducted by Mitton et al in 2007 which revealed that the key knowledge transfer and exchange strategies between researchers and policymakers and highlighted by the studied literature were face-to-face-exchange, consultations and regular meetings; networks and communities of practice; and steering committees to integrate views of local experts into design, conduct and interpretation of research. ${ }^{17}$ The Advisors also stressed that it is good to have a program of funded research that informs policymaking. They thought that this can help in setting up priorities and plan research based on these priorities. In addition to that, the availability of financial support will guarantee regular research conduction based on real needs, and researchers will be more motivated and ready to conduct research without financial constraints. These results agree with those of the Uganda study in 2012 which tackled the perspectives of policy actors on improving the uptake of evidence in health policy development and implementation, and revealed that twenty of the reviewed papers included in this study emphasized the availability of funding for undertaking research activities as one of the important specific facilitating factor for evidence-based policymaking. ${ }^{18}$ The advisors also thought that policymaking department should have specific scientific advisors on individual issues since these advisors can contribute in enhancing the translation of knowledge into practice through the use of research evidence in strategic planning, and that their 
experience can be utilized in a better choosing, implementing and supervising of relevant researches. The policymakers who participated in the study conducted by Hyder et al, also suggested establishing an office composed of specialists who will be able to look at those disseminated research results, critically analyze them and advise government and the appropriate ministry. ${ }^{16}$

\section{Conclusion}

There is a poor role of research in health policymaking in Iraqi Kurdistan as policymakers very rarely consult researchers in their decision making. There is also poor networking among researchers, policy-makers, practitioners and representatives of civil society which has its negative impact on evidence-based policymaking. There is obviously a lack of any sort of programme of funded research that can inform policymaking. A culture of collaboration between policymakers and researchers needs to be encouraged and this could be done through initiating co-ordination planning networks and establishing communication channels between these two groups. Academic researchers need to prepare reports, with simple and understandable language, on their research work for relevant policymakers. Further research work needs to be planned in this regard for further exploration of this important issue.

\section{Conflicts of interest}

The authors report no conflicts of interest.

\section{References}

1. Davies P. 'Is evidence-based government possible?' Jerry Lee Lecture, presented at the $4^{\text {th }}$ Annual Campbell Collaboration Colloquium, Washington DC; 2004.

2. Sutcliffe S, Court J. A toolkit for progressive policymakers in developing countries. Research and Policy in Development Programme. 111 Westminster Bridge Road, London, SE1 7JD; 2006.

3. Swartz L, Tomlinson $M$, Landman $M$. Evidence, policies and practices: Continuities and discontinuities in mental health promotion in a developing country. Int $\mathrm{J}$ Ment Health Prom 2004; 6:33-7.
4. Kothari A, Birch S, Charles C. "Interaction" and research utilization in health policies and programs: does it work? Health Policy 2005; 71:117-25.

5. Innvaer S, Vist $G$, Trommald M, Oxman A. Health policy-makers' perceptions of their use of evidence: a systematic review. J Health Serv Res Policy 2002; 7:239-44.

6. Lavis $\mathrm{J}$, Davies $\mathrm{H}$, Oxman A, Denis $\mathrm{J}-\mathrm{L}$, Golden-Biddle K, Ferlie E. Towards systematic reviews that inform health care management and policy-making. J Health Serv Res Policy 2005; 10:35-48.

7. Lawis JN, Ross S, Hurley JE, Hohenadel JM, Stoddart JL, Woodward CA, et al. Examining the role of Health Services Research in Public Policymaking. Milbank Q 2002; 80(1):125-54.

8. Tawfik-Shukor A, Khoshnaw H. The impact of health system governance and policy processes on health services in Iraqi Kurdistan. BMC Int Health Hum Rights 2010; 10:14.

9. Abdulahad F, Shabila NP. A perception-based survey on evaluating the impact of locally published medical journals. Middle East J Fam Med 2009; 7(9):31-4.

10. Shabila NP, Al-Taweel N, Tahir R, Shwani FH, Salih AM, Al-Hadithi TS. Iraqi health system in Kurdistan region: medical professionals' perspectives on challenges and priorities for improvement. Confl Health 2010; 4:19.

11. Europea Commision. Scientific evidence for policy-making. European research area, socio-economic sciences and humanities 2008:25-7. Available from: http://ec.europa.eu/ research/research-eu [Accessed on 17 March 2014]

12. Uneke CJ, Ezeoha AE. Development of Health Policy and Systems Research in Nigeria: Lessons for Developing countries 'Evidence-Based Health Policy Making Process and Practice. Healthcare Policy 2010; 6(1): 109-26.

13. Feldman $\mathrm{PH}$, Nadash $\mathrm{P}$, Gursen $\mathrm{M}$. Improving communication between researchers and policymakers in long-term care: or, Reesearchers are from Mars; Policymakers are from Venus. The Gerontologist 2001; 41 (3):312-21

14. El-Jardali F, Lavis JN, Ataya N, Jamal D. Use of health systems and policy research evidence in the health policymaking in eastern Mediterranean countries: views and practices of researchers. Implementation Science 2012; $7: 2$.

15. Nutley S. 'Bridging the Policy/Research Divide: Reflections and Lessons from the UK', Keynote Paper at National Institute of Governance Conference 'Facing the Future: Engaging stakeholders and citizens in developing public policy', Canberra, Australia; 23-4 April 2003. 
16. Hyder AA, Corluka A, Winch PJ, El-Shinnawy A, Ghassany $H$, Malekafzail $H$, et al. National policy-makers speak out: are researchers giving them what they need? Health Policy Plan 2011; 26:73-82

17. Mitton C, Adair CE, McKenzie E, Patten SB, Waye PB. Knowledge transfer and exchange: review and synthesis of the literature. The Milbank Quarterly 2007; 85(4):729-68.

18. WHO. Proposed networks to support health decision-making and health policy formulation in low and lower middle income countries and considerations for implementation. Geneva: World Health Organization; 2005. 